\title{
Aspetti ecologici ed evolutivi del popolamento di grotte temperate e tropicali: Osservazioni sul ciclo biologico di alcune specie di Ptomaphagus (Coleoptera Catopidae)*
}

\author{
di \\ Valerio SBORDONI e Marina COBOLLI-SBORDONI**
}

Il popolamento animale di grotte situate in regioni tropicali ha suscitato negli ultimi anni l'interesse di molti ricercatori. In vari paesi sono state organizzate campagne di ricerca e spedizioni biospeleologiche che hanno portato un notevole contributo alla conoscenza del popolamento ipogeo tropicale. Ricordiamo, scegliendo tra le più recenti, la missione zoologica belga alle Galapagos e in Equador (Leleup, 1968; Vari Autori, 1968, 1970), la spedizione biospeleologica rumena a Cuba (Botosaneanu, 1970; Decou et al. 1971), le ricerche della Association for Mexican Caves Studies (Reddell \& Mitchell, 1971), le spedizioni zoologiche italiane in Messico (Sbordoni \& Argano, 1972; Vari Autori, 1972; Sbordoni et al., 1973) e le ricerche dei francesi nella grotta di Sof Omar in Etiopia (Monod \& Morton, 1972).

I risultati di queste ricerche, realizzate per lo più da equipes organizzate rivolte allo studio di tutta la fauna piuttosto che di singoli gruppi sistematici ci consentono ora di avere le idee più chiare sulla composizione delle speleofaune tropicali e sulla ecologia delle cavità sotterranee.

Oggetto di questa comunicazione è quello di discutere le differenze ecologiche esistenti tra le grotte temperate e quelle tropicali e di esaminarne la relazione con l'evoluzione dei troglobi, sulla base di ricerche da noi condotte su coleotteri Catopidi del genere Ptomaphagus di grotte messicane. In particolare i risultati delle nostre indagini concordano con l'idea che l'elevato imput di energia nelle grotte tropicali indebolisce le pressioni selettive che controllano l'adattamento dei cavernicoli a favore di un risparmio di energia e che quindi la velocità di evoluzione dei troglobi è più lenta nelle grotte di tipo tropicale che in quelle di tipo temperato.

$\mathrm{Da}$ un punto di vista ecologico le grotte tropicali differiscono notevolmente da quelle temperate. La differenza più ovvia riguarda la temperatura. Mentre la media delle grotte europee è compresa, a seconda della quota e della latitudine, $\operatorname{tra} 8^{\circ} \mathrm{e}$ $15^{\circ} \mathrm{C}$, quella delle grotte tropicali si aggira sui $25^{\circ}$. Temperature più elevate si possono riscontrare in grotte abitate da fitte colonie di chirotteri. In Chiapas abbiamo esplorato una grotta, la Cueva de la Ramillette, con una temperatura di $32^{\circ}$ (Sbordoni et al., 1973) e a Cuba è segnalata una grotta dove la temperatura raggiunge i $37^{\circ}$ (Decou et al., 1971). Sembra che in questi casi il clima sia fortemente influenzato dalle enormi colonie di Phyllostomidae presenti nelle grotte.

\footnotetext{
* Comunicazione presentata al Simposio sugli "Artropodi di caverna" nel IX Congresso Italiano di Entomologia, Siena 21-25 giugno 1972.

** Istituto di Zoologia dell' Università, Viale dell'Università 32, 00100 Roma-Italia.
} 
Maggiori differenze si niscontrano nelle composizione delle comunità cavernicole. Chi visita per la prima volta una grotta tropicale rimane sorpreso dalla quantità numerica di individui e dalla eterogeneità del popolamento. Quello che in particolare sorprende è l'abbondanza di avventizi, di forme animali che, non mostrando alcun carattere particolare di adattamento alla vita cavernicola, pure si sono insediate con successo nella grotta tropicale, svolgendovi per intero il proprio ciclo vitale. Questa osservazione vale a tutti i livelli trofici: dalla microfauna guanobia ai predatori vaganti. In pratica l'indice di diversità delle communità cavernicole tropicali è considerevolmente più elevato di quello delle comunità cavernicole temperate. Questa situazione appare legata alla maggiore quantità di energia stabilmente disponibile in una grotta tropicale.

Il guano rappresenta la fonte di energia di gran lunga più importante nelle grotte tropicali, dove le colonie di chirotteri possono raggiungere facilmente le decine e centinaia di migliaia di individui. La struttura delle catene alimentari in una grotta a guano può essere estremamente articolata: McClure et al. (1967) osservano che nella sola Dark Cave, nelle Batu Caves in Malesia, vivono almeno 174 specie di animali, inclusi 23 specie di vertebrati. Horst (1972) mostra che anche in una grotta artificiale come una miniera abbandonata si può insediare una comunità cavernicola complessa che si sviluppa esclusivamente sulla energia fornita dal guano.

Harris (1970) discute altri aspetti delle grotte a guano e mette in evidenza una varietà di condizioni ambientali che si succedono nel corso dell'anno, sottolineando il carattere decisamente stagionale delle comunità animali in alcune grotte. Tutto ciò contrasta in modo netto con la situazione che si verifica nelle caverne di tipo temperato dove le colonie di chirotteri sono generalmente ridotte o assenti, il clima molto più stabile e le risorse trofiche generalmente scarse e sporadiche.

Differenze rilevanti riguardano infine la varietà delle risorse trofiche che derivano dal guano: nelle grotte tropicali coesistono frequentemente specie di chirotteri a regime carnivoro, piscivoro, insettivoro, ematofago, frugivoro, nettarivoro. Nelle grotte di regioni temperate troviamo invece quasi esclusivamente insettivori. La diversità del guano che risulta dai diversi ruoli trofici dei pipistrelli determina nelle grotte tropicali una considerevole estensione delle nicchie utilizzabili. Un tentativo di analisi quantitativa dei ruoli trofici dei chirotteri nelle varie regioni zoogeografiche è stato recentemente effettuato da Wilson (1973).

Se esaminiamo la distribuzione geografica dei cavernicoli possiamo inoltre osservare, almeno nelle linee generali, che gli organismi troglobi sono più abbondanti nelle regioni temperate. Nelle grotte tropicali la fauna troglobia terrestre é più scarsa e la maggioranza dei troglobi è rappresentata da organismi acquatici.

Mitchell (1969) discute questa situazione riproponendo una spiegazione al problema della "evoluzione regressiva" o rudimentazione strutturale degli animali cavernicoli, tuttora oggetto di controversie da parte dei biologi (vedi Barr, 1968, e Wilkens, 1973). In pratica egli ammette negli organismi di grotte temperate una velocità di evoluzione maggiore che in organismi di grotte tropicali. Questa diversa velocità di evoluzione sarebbe dovuta alle più forti pressioni selettive che operano in 
condizioni di scarsa disponibilità trofica. Come ha mostrato Heuts (1953) molti caratteri regressivi tipici dei troglobi come la riduzione dell'occhio e del pigmento possono essere selettivamente vantaggiosi in termini di economia metabolica e di maggiore efficienza nella utilizzazione dell'energia disponibile.

Questa tesi mette l'enfasi sul fattore trofico quale principale agente selettivo nell'adattamento alla vita cavernicola, e dà una spiegazione plausibile in termini neodarwiniani dell'evoluzione regressiva dei cavernicoli ponendo in secondo piano l'influenza dei fattori abiotici, ma purtroppo poggia su basi ancora incerte.

In primo luogo la presenza di troglobi terrestri in alcune grotte tropicali rappresenta un problema sconcertante. La situazione per i ragni è stata esaminata da Brignoli (1973) in questo simposio: i ragni ciechi a facies apparentemente troglobia sono tutt'altro che rari in grotte tropicali. Le nostre ricerche in Messico (Sbordoni et al., 1973) hanno permesso di scoprire varie forme cieche a facies troglobia di Carabidi (Anillini), Isteridi, Tisanuri, Diplopodi, Ricinulei e Ragni in grotte a carattere decisamente tropicale. Le ricerche di Leleup alle Galapagos hanno portato alla luce numerosi elementi ciechi tra cui addirittura un Tenebrionide. La forma più specializzata di Scorpione cavernicolo è stata rinvenuta recentemente in una grotta calda messicana (Mitchell, 1971) e molti altri esempi potrebbero aggiungersi.

In secondo luogo i dati disponibili attualmente per un confronto accurato tra popolamenti temperati e tropicali sono ancora troppo scarsi. Mentre la fauna cavernicola di alcune regioni dell'Europa è degli Stati Uniti sono conosciute a un livello soddisfacente per molti gruppi tassonomici, le conoscenze su molte aree tropicali richiedono un ulteriore approfondimento. Per questa insufficienza di dati è scarsamente indicativo un confronto tra i due tipi di popolamento almeno nella maggioranza dei taxa.

Una terza difficoltà sorge a livello geografico: esistono buone messe a punto di faune cavernicole tropicali, ma di singole aree lontane tra loro, appartenenti a regioni zoogeografiche distanti e un confronto con i popolamenti temperati risulta solo indicativo. Lo stesso Mitchell a sostegno della propria tesi paragona la fauna cavernicola della Sierra del Abra in Messico con quella dello Edwards Plateau in Texas, utilizzando i risultati in modo piuttosto superficiale. In questa analisi egli calcola complessivamente 59 specie troglobie nell'Edwards Plateau contro 6 nella Sierra del Abra, senza tener conto che queste cifre sono considerevolmente influenzate dai processi di speciazione che possono aver determinato una moltiplicazione delle specie troglobie di un dato genere in una regione e non nell'altra, come di fatto avviene in alcuni Coleotteri (Rhadine), Ragni ed Opilioni dell'Edwards Plateau. In tal caso il numero di specie troglobie dipende più dalle condizioni dell'area, che possono aver favorito l'isolamento di popolazioni cavernicole, che non dalle diverse pressioni selettive. Inoltre il livello di conoscenza faunistica delle due aree era considerevolmente diverso nel 1969 essendo la fauna del Texas conosciuta e studiata da più tempo. Negli ultimi tre anni dal 1970 al 1973 la fauna troglobia della Sierra del Abra ha infatti raddoppiato il numero di troglobi terrestri (Reddell \& Mitchell, 1971; Shear, 1973; Vomero, 1973), e le fiorenti ricerche in corso nell'area fanno prevedere un ulteriore aumento. In conclusione l'ipotesi di Mitchell mostra un considerevole interesse speculativo, ma poggia su evidenze sperimentali ancora insufficienti. 
Tali difficoltà possono essere in buona parte superate studiando il popolamento di grotte con caratteristiche climatiche diverse, situate in una medesima area limitando l'analisi a generi e specie presenti in entrambi i tipi di cavità. Nel 1969 abbiamo potuto esaminare una serie di grotte nel Tamaulipas e San Luis Potosi (Messico) che vanno da $300 \mathrm{~m}$ s.l.m. fino a $2300 \mathrm{~m}$. Nel 1971 abbiamo potuto esplorare in Chiapas grotte, situate a poche decine di $\mathrm{Km}$ di distanza, alle più diverse quote. Da grotte tropicali che si aprono in piena foresta pluviale, a $100 \mathrm{~m} \mathrm{di}$ quota, fino a grotte decisamente temperate, in bosco di conifere ad oltre $2400 \mathrm{~m}$. I risultati di queste ricerche finanziate dall'Accademia Nazionale dei Lincei e realizzate dagli Istituti di Zoologia di Roma e Milano, effettuate in una cinquatina di cavità, sono tuttora allo studio o in corso di pubblicazione.

In base alle ricerche effettuate è risultato che i Coleotteri Catopidi del genere Ptomaphagus sono tra i non molti animali in grado di popolare entrambi i tipi di cavità, e quindi costituiscono un materiale estremamente favorevole per valutare l'adattamento alle due diverse condizioni. Abbiamo osservato colonie di questi Catopidi in molte delle grotte visitate e lo studio tassonomico che uno di noi sta effettuando ha portato al riconoscimento di alcune nuove entità. Nelle grotte americane gli Ptomaphagus occupano una nicchia ecologica per molti versi analoga a quella dei nostri Bathysciinae; si tratta di animali a regime saprofago, che frequentemente utilizzano il guano a scopo alimentare.

\section{MATERIALE E METODI}

Per la presente ricerca abbiamo utilizzato 3 specie di Ptomaphagus. La prima mostra facies troglobia, si tratta di Ptomaphagus troglomexicanus, descritto recentemente su un unico individuo della Cueva de la Perra, nella Sierra de Guatemala, Tamaulipas, (Peck, 1968). Questa cavità si trova a 2200 metri di quota, in foresta mista, ha una temperatura all'interno di $11,5^{\circ} \mathrm{C}$, è priva di colonie di Chirotteri ed ha le caratteristiche tipiche di una grotta temperata. Con l'aiuto di esche abbiamo potuto raccogliervi il 25 novembre 1969 un centinaio di individui che sono stati portati a Roma e allevati in laboratorio.

Della seconda specie, Ptomaphagus spelaeus, furono prelevati un centinaio di individui il 28 ottobre 1969 nella parte terminale della grotta di Cacahuamilpa, a $1200 \mathrm{~m}$, nello stato di Guerrero dove si registrò una temperatura di $22^{\circ}$. Anche questa specie, endemica delle grotte a guano della zona di Cacahuamilpa, tutte a carattere decisamente tropicale, era considerata troglobia.

Abbiamo utilizzato queste due specie, molto vicine filogeneticamente per un confronto dettagliato morfologico, ecologico e fisiologico con l'intento di saggiare eventuali differenze nel grado di adattamento alla vita troglobia e nella rudimentazione strutturale. In questo esame abbiamo aggiunto per confronto Ptomaphagus pius specie non cavernicola dell'Italia centrale. Allevamenti di questa specie sono stati effettuati più volte nel nostro Istituto a partire dal 1967 con materiale raccolto con esche alla Bufalotta, negli immediati dintorni di Roma. 
I caratteri presi in considerazione sono:

1. sviluppo relativo di arte e appendici.

2. grado di riduzione dell'occhio.

3. grado di riduzione del pigmento.

4. grado di riduzione alare.

5. grado di specializzazione delle strutture sensoriali del $7^{\circ}, 9^{\circ}$ e $10^{\circ}$ antennomero (organo antennale). (vedi Baccetti e Sbordoni, 1967 e Peschillo, 1970).

6. dimensioni delle uova.

7. durata dei vari stadi del ciclo biologico.

Gli allevamenti sono stati realizzati, secondo una metodica da noi messa a punto (Sbordoni e Cobolli, 1969), in camere termostatate, in vaschette con gesso, argilla e guano. Gli animali sono stati allevati a temperature simili a quelle registrate nei biotopi d'origine: $14^{\circ} \mathrm{C}$ per Pt. troglomexicanus. $22^{\circ} \mathrm{C}$ per Pt. spelaeus e $19^{\circ} \mathrm{C}$ per Pt. pius. Controlli sono stati effettuati allevando Pt. spelaeus anche a $19^{\circ} \mathrm{C}$ e Pt. pius anche a $22^{\circ} \mathrm{C}$. La temperatura non ha influito sensibilmente sulla durata del ciclo.

\section{RISULTATI}

Nella tabella 1 vengono presentati in sintesi i risultati dell'esame morfologico. Si può innanzitutto osservare una correlazione tra lo sviluppo relativo delle antenne e la regressione del pigmento e dell'occhio nelle tre specie esaminate.

\begin{tabular}{|c|c|c|c|}
\hline Tabella 1 & $\begin{array}{l}\text { Pt. pius } \\
\text { (umicolo } \\
\text { Italia) }\end{array}$ & $\begin{array}{l}\text { Pt. spelaeus } \\
\text { (cavernicolo } \\
\text { grotta } \\
\text { tropicale }\end{array}$ & $\begin{array}{l}\text { Pt. troglo- } \\
\text { mexicanus } \\
\text { (cavernicolo } \\
\text { grotta } \\
\text { temperamenta) }\end{array}$ \\
\hline $\begin{array}{l}\text { Lunghezza antenna } \\
\text { lunghezza totale }\end{array}$ & 0,31 & 0,35 & 0,55 \\
\hline $\begin{array}{l}\text { Diametro orizzontale } \\
\text { dell'occhio } \\
\text { larghezza del capo }\end{array}$ & 0,92 & 0,84 & 0,37 \\
\hline Pigmento & $\begin{array}{l}\text { normalmente } \\
\text { pigmentato }\end{array}$ & $\begin{array}{l}\text { pigmento } \\
\text { ridotto }\end{array}$ & $\begin{array}{l}\text { pigmento } \\
\text { molto ridotto }\end{array}$ \\
\hline Riduzione alare & $\begin{array}{l}\text { dimorfismo: } \\
\text { macrotteri e } \\
\text { brachitteri }\end{array}$ & macrottero & attero \\
\hline $\begin{array}{l}\text { Organo antennale } \\
7^{\circ} \text { antennomero } \\
(2 \text { camere sensorie })\end{array}$ & $\begin{array}{l}\text { circa } 18 \\
\text { sensilli }\end{array}$ & $16-18$ sensilli & $\begin{array}{l}\text { oltre } 50 \\
\text { sensilli }\end{array}$ \\
\hline $\begin{array}{l}\text { Organo antennale } \\
9^{\circ} \text { e } 10^{\circ} \text { antennomero }\end{array}$ & $\begin{array}{l}2+2 \text { fossette } \\
\text { rudimentali } \\
\text { sensorie }\end{array}$ & $\begin{array}{l}2+2 \text { fossette } \\
\text { rudimentali } \\
\text { sensorie }\end{array}$ & $\begin{array}{l}2+2 \text { camere } \\
\text { sferiche, } \\
\text { completamen- } \\
\text { te invaginate }\end{array}$ \\
\hline Dimensioni dell'uovo in $\mu$ & $650 \times 600$ & $900 \times 600$ & $1200 \times 800$ \\
\hline
\end{tabular}




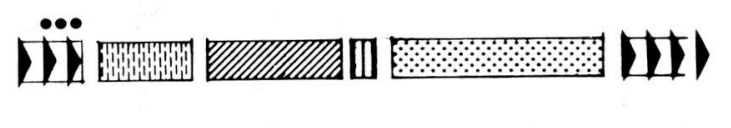

E.watsonl

17.1.

\section{Pt.plus}

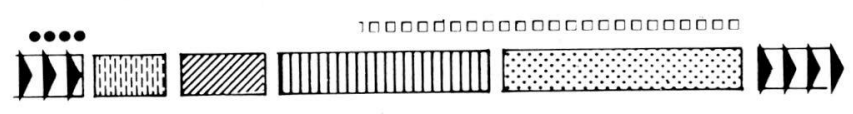

III imago

牂 Letà larvale

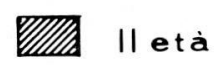

IIIIIII Illetà

pupa

$\bullet \bullet \bullet \quad$ ovo

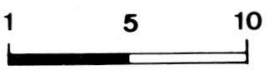

giorni

๑ง celletta d'argilla

pt. spelaeus

.i.i.

П ח III della pupa. 
$P$. troglomexicanus appare morfologicamente la più specializzata e per lo sviluppo realtivo delle antenne e per la complicazione strutturale degli organi a probabile funzione chemiorecettrice, presenti nel $7^{\circ}, 9^{\circ}$ e $10^{\circ}$ segemento dell'antenna. In tutti gli Ptomaphagini esistono due camere sensorie nel $7^{\circ}$ segmento antennale. In particolare il numero di cellule sensoriali presenti nella camera sensoriale più sviluppata nel $7^{\circ}$ antennomero supera i 50 sensilli raggiungendo un grado di complicazione che trova riscontro solo nelle forme più specializzate di Bathysciinae dei generi Leptodirus e Astagobius (Sbordoni, dati inpubblicati,

Peschillo, 1970). J_o sviluppo delle camere sensorie del $7^{\circ}$ antennomero nelle altre due specie è notevolmente ridotto e analoga è la situazione delle strutture sensoriali nel $9^{\circ}$ e $10^{\circ}$ antennomero: soltanto $P$. troglomexicanus mostra in ciascuno di questi segmenti 2 camere sensoriali sferiche, completamente invaginate e composte da numerosi sensilli. In $P$. spelaeus e $P$. pius si osservano due fossette semisferiche poco profonde con pochi sensilli tricoidi e bastoncellari, simili a quelle che si trovano negli stessi antennomeri di molti Catopidi di superficie dei generi Catops, Choleva e Nargus (Sbordoni, dati inpubblicati, Peschillo, 1970).

Anche nell'analisi delle strutture soggette a rudimentazione come occhi, pigmento, ali troviamo notevole somiglianza tra $P$. pius e $P$. spelaeus da una parte e $P$. troglomexicanus dall'altra. In $P$. spelaeus, cavernicolo di grotta tropicale, l'occhio è normalmente sviluppato, di poco più ridotto che in $P$. pius, epigeo. In $P$. troglomexicanus si assiste invece a una notevole riduzione dell'area oculare con pochi ommatidi privi di pigmento.

Le ali metatoraciche sono assenti in $P$. troglomexicanus, dove si osservano soltanto dei rudimenti, mentre sono completamente sviluppate in P. spelaeus. In Ptomaphagus pius troviamo sia popolazioni macrottere che popolazioni con dimorfismo alare. La popolazione della Bufalotta (Roma) è pterodimorfica: accanto a individui macrotteri con ala normalmente sviluppata troviamo forme brachittere con notevole riduzione clella porzione distale. L'ala degli individui brachitteri presenta una notevole riduzione delle venature mediana, radiale e della porzione distale della cubitale. Su 33 individui esaminati 18 erano brachitteri e 15 macrotteri. Il

\begin{tabular}{|c|c|c|c|}
\hline Tabella 2 & $\begin{array}{l}\text { Pt. pius } \\
\text { (umicolo } \\
\text { Italia) }\end{array}$ & $\begin{array}{l}\text { Pt. spelaeus } \\
\text { (cavernicolo } \\
\text { grotta } \\
\text { tropicale) }\end{array}$ & $\begin{array}{l}\text { Pt. troglo- } \\
\text { mexicanus } \\
\text { (cavernicolo } \\
\text { grotta } \\
\text { temperata) }\end{array}$ \\
\hline $\begin{array}{l}\text { Uovo } \\
\text { Larva I êtà } \\
\text { Larva II età } \\
\text { Larva III età } \\
\text { Pupa } \\
\text { Adulto } \\
\text { Durata complessiva } \\
\text { media del } \\
\text { ciclo preimaginale }\end{array}$ & $\begin{array}{l}\text { 2-4 giorni } \\
3 \text { giorni } \\
8 \text { giorni } \\
2-3 \text { giorni } \\
16 \text { giorni } \\
\text { circa } 2 \text { mesi } \\
30 \text { giorni }\end{array}$ & $\begin{array}{l}3-4 \text { giorni } \\
1-4 \text { giorni } \\
2-5 \text { giorni } \\
8-10 \text { giorni } \\
10 \text { giorni } \\
\text { circa } 2 \text { mesi } \\
28 \text { giorni }\end{array}$ & $\begin{array}{l}\text { 8-11 giorni } \\
6-10 \text { giorni } \\
4-6 \text { giorni } \\
\text { 17-25 giorni } \\
\text { circa } 30 \text { giorni } \\
\text { oltre } 1 \text { anno } \\
\text { in laboratorio } \\
73 \text { giorni }\end{array}$ \\
\hline
\end{tabular}


brachitterismo sembra più frequente nei maschi. Su 18 individui brachitteri 14 , pari all' $83 \%$, erano maschi.

La tabella 2 mette a confronto la durata dei vari stadi del ciclo biologico nelle tre specie. Anche in questo caso è da rilevare la somiglianza del ciclo biologico in $P$. pius e $P$. spelaeus. Per contro $P$. troglomexicanus mostra un notevole allungamento del ciclo preimaginale, che supera considerevolmente non solo la durata di quello delle altre 2 specie studiate, ma anche quella di $P$. loedingi, troglobio dell'Alabama, studiato da Peck (1967). Dai dati di questo autore risulta una durata media del ciclo preimaginale di 42 giorni. In ciascuna delle tre specie da noi studiate è stata osservata costruzione di celleta d'argilla da parte delle larve di $3^{\circ}$ età. La fig. 1 visualizza queste differenze; per un confronto è stata aggiunta una specie epigea di Catopide: Sciodrepoides watsoni, da noi precedentemente allevata in laboratorio. La morfologia delle larve verrà esaminata in altra sede; qui si può osservare che nella specie a ciclo lungo, $P$. troglomexicanus, non si osservano rudimentazioni paragonabili a quelle riscontrate nei Bathysciinae da Deleurance-Glaçon (1963). L'uovo è notevolmente più grande in $P$. troglomexicanus e il numero di uova prodotte è inferiore a quello delle altre 2 specie.

\section{DISCUSSIONE E CONCLUSIONI}

Questi dati consentono vari ordini di considerazioni. In primo luogo è evidente il parallelismo che risulta con il ciclo biologico della sottofamiglia Bathysciinae, studiata dalla Deleurance. Si può osservare, ad esempio, che mentre l'allungamento del ciclo dei Batiscini specializzati si accompagna sempre a una contrazione delle età larvali (che in alcune specie si riducono a una sola: la prima), in Ptomaphagus si attua in maniera opposta un aumento della durata in tutti gli stadi. Inoltre negli Ptomaphaginae la costruzione della celletta di argilla non sembra legata all'evoluzione verso la vita troglobia, ma sembra un carattere biologico tipico della sottofamiglia.

Ma qui ci interessa soprattutto mettere in luce l'elevata specializzazione di $P$. troglomexicanus, rispetto alle altre due specie, riscontrabile a tutti i livelli e soprattutto nella durata del ciclo (carattere associato alla generale riduzione del metabolismo) e nella specializzazione sensoriale. Si rileva inoltre che la rudimentazione delle ali, dell'occhio e del pigmento accompagna di pari passo lo sviluppo delle strutture "positive" nell'adattamento alla vita cavernicola, così che $P$. troglomexicanus, che è la più specializzata, è anche la specie che mostra in maggior misura rudimentazione strutturale. La specie cavernicola tropicale, $P$. spelaeus, pur essendo molto affine filogeneticamente alla precedente (Peck, 1968; Sbordoni, in preparz.) ne differisce profondamente per il livello di specializzazione e non mostra nei confronti di $P$. pius, epigeo, quelle differenze che ci si potrebbero aspettare dalla diversa ecologia delle due specie. Entrambe le specie cavernicole discendono da un antenato epigeo non dissimile nel livello di adattamento da $P$. pius, e hanno verosimilmente colonizzato le grotte contemporaneamente, in connessione con l'inaridimento climatico del postglaciale. 
Questi dati sono in accordo con l'ipotesi precedentemente discussa che prevede diverse velocità di evoluzione in popolazioni cavernicole temperate e tropicali dovute alle differenti pressioni selettive che operano nei due tipi di grotte.

Gli stessi dati non sembrano invece accordarsi con la teoria che attribuisce alla pressione di mutazioni e in ultima analisi al tempo il ruolo responsabile delle differenze del grado di rudimentazione strutturale (Wilkens, 1973). Se così fosse bisognerebbe ammettere ondate successive di colonizzazione, a partire da specie ancestrali epigee diverse, in un gruppo di specie che all'analisi sistematica risulta estremamente compatto filogeneticamente; cioè non ci sono motivi per ritenere che $P$. troglomexicanus sia un cavernicolo più antico di $P$. spelaeus.

\section{RINGRAZIAMENTI}

La maggior parte del materiale utilizzato nel presente studio è stato raccolto nel corso di missioni in Messico finanziate dall'Accademia Nazionale dei Lincei e promosse dal prof. P. Pasquini. Ringraziamo il Dr. Roberto Argano compagno nelle raccolte in Messico e il Sig. Vincenzo Vomero che raccolse parte del materiale di Ptomaphagus pius utilizzato negli allevamenti.

\section{RIASSUNTO}

Scopo di questa ricerca è un controllo sperimentale di una teoria, di recente avanzata, volta a spiegare la scarsezza di troglobi terrestri in grotte tropicali (ricche di risorse trofiche) rispetto alle grotte temperate (più povere di risorse) come una conseguenza delle minori pressioni selettive favorenti gli adattamenti tipici dei troglobi (rallentamento del metabolismo ecc.) nelle grotte del primo tipo.

E' stato studiato il grado di adattamento alla vita ipogea di due specie affini messicane di Ptomaphagus, probabilmente derivate da un medesimo antenato epigeo. Di queste $P$. troglomexicanus Peck vive in una tipica grotta temperata fredda, d'alta quota, con scarse risorse trofiche nella Sierra de Guatemala (Tamaulipas), mentre $P$. spelaeus (Bilimek) vive in grotte tropicali calde, di bassa quota, con abbondanti risorse alimentari nel Guerrero; un paragone è anche fatto con $P$. pius Seidlitz, specie epigea dell'Europa meridionale.

Tra $P$. troglomexicanus e le altre due specie vi sono cospicue differenze sia morfologiche (lunghezza relativa delle antenne, complessità strutturale dell'organo antennale, riduzioni degli occhi, delle ali e della pimentazione) che fisiologiche (lunghezza del ciclo vitale). Queste differenze sembrano dovute alle differenti pressioni selettive operanti nei due tipi di grotte. Scarse sono invece le differenze tra $P$. spelaeus e $P$. pius.

Questi fatti sono a favore dell'esistenza di una relazione tra tasso di evoluzione ed ecologia delle grotte (e più in particolare delle risorse trofiche presenti) e non dell'ipotesi di una neutralità dal punto di vista selettivo delle mutazioni favorenti processi degenerativi. 


\section{SUMMARY}

Differences between tropical and temperate cave communities are an important topic in the actual biospeleological thinking. Among the most striking differences is the paucity of terrestrial troglobites in tropical caves. This fact may depend on the higher energy input into tropical caves which lessens the selection pressures for energy-economizing troglobite adaptations. Consequently evolutionary rates would be slowed in tropical caves and, in a date group, troglobites would appear later in such caves than in temperate ones with lower energy input.

In order to investigate this point the authors studied the degree of adaptation to the cave environment in two species of Mexican Ptomaphagus which, being phylogenetically related, probably descend from the same epigean ancestor. Among these species the first one, $P$. troglomexicanus Peck, lives in a typical temperate cave (i.e. cold, high altitude cave, with scarce food supply) in the Sierra de Guatemala (Tamaulipas), the other one, P. spelaeus (Bilimek), populates tropical caves (i.e. warm, lowland cave, with rich food supply) in the State of Guerrero. In addition a comparison is made with $P$. pius Seidlitz, an epigean species from southern Europe.

The results show a striking difference between $P$. troglomexicanus on a side and the other two species. Differences chiefly concern morphological features such as relative antenna length, structural complexity (i.e. the number of sensilla) of the antenna chemioreceptor organs in the $7^{\circ}, 9^{\circ}, 10^{\circ}$ segments, degree of reduction of eye, wing and pigmentation and physiological ones such as the length of the life cycle. The possible causes of these differences are discussed. According the authors these differences appear due to the different selection pressures acting in the two types of caves. In addition a comparison between the "tropical cave" species, $P$. spelaeus, with the epigean one, $P$. pius, does not point out the differences that one could expect by the diverse ecology of these species.

These observations support the idea that evolutionary rates in cavernicoles are strongly affected by the ecology of the cave, mainly depending on the degree of energy input, and are poorly consistent with the hypotesis that mutations affecting degenerative processes are selectively neutral.

\section{BIBLIOGRAFIA}

BACCETTI, B. e V. SBORDONI. 1967 - Prime osservazioni ultrastrutturali sull'organo antennale dei Bathysciinae. Boll. Zool., 34, Atti XXXIV Conv. U.Z.I.: 84 - 85.

BARR, Th.C. jr., 1968 - Cave Ecology and the Evolution of Troglobites. In "Evolutionary Biology" II. Dobzhansky, Hecht and Steere eds., Appleton - Century - Crofts, New York: $35-102$.

BOTOSǍNEANU, L., 1970 - L'expédition biospéologique à Cuba (mars-juin 1969) organisée par les Académies des Sciences de Cuba et de Roumanie: présentation sommaire des stations explorées pour le prélèvement d'échatillons de faune aquatique souterraine. Trav. Inst. Spéol. "Emile Racovitza", 9: 81 - 95.

BRIGNOLI, P.M., 1973 - Il popolamento di Ragni nelle grotte tropicali. Int. J. Speleol.: 5: $325-336$. 
DECOU, V.Gh., St. NEGREA, Gh. RACOVITZA et C. FUNDORA MARTINEZ, 1971 Première expédition biospéologique cubano-roumaine à Cuba (1969). Quelques observations sur les stations terrestres prospectées. Trav. Inst. Spéol. "Emile Racovitza", 10:71-79.

DELEURANCE-GLACON, S., 1963 - Recherches sur les coléoptères troglobies de la sousfamille des Bathysciinae. Ann. Sci. Nat. (Zool.), (12) 5 (1): $1-172$.

FAGE, L., 1931 - Biospeologica. LV. Araneae. Arch. de Zool. Exp. et Gén., 71: 99 - 291.

HARRIS, J.A., 1970 - Bat guano cave environment. Science, 169: 1342 - 1343.

HEUTS, M.J., 1953 - Regressive evolution in cave animals. Sympos. Soc. Exp. Biol., Evolution, 7: $290-309$

HORST, R., 1972 - Bats as primary producers in an ecosystem. Bull. Nat. Speleological Soc., $34(2): 49-54$.

LELEUP, N., 1968 - Introduction. In "Mission zoologique belge aux îles Galapagos et en Ecuador", I, Mus. R. Afrique Cen. et Inst. R. Sc. Nat. Belg., Bruxelles: $9-34$.

LELEUP, N., 1971 - Origin and evolution of holarctic and intertropical cave fauna. Soc. Suisse Spel. Actes 4 Cong. Nat. Spel., Supp. Stalactite, 6: $199-204$.

McClURE, H.E., B. LIM, S.E. WINN, 1967 - Fauna of the Dark Cave, Batu Caves, Kuala Lumpur, Malaysia. Pac. Insects, 9: $399-428$.

MITCHELL, R.W., 1969 - A comparison of temperate and tropical cave communities. The Southwestern Naturalist, 14 (1): $73-88$.

MITCHELL, R.W., 1971 - Typhlochactas elliotti, a new eyeless cave scorpion from Mexico (Scorpionida, Chactidae). Ann. Spéléol., 26 (1): 135 - 148.

MONOD, Th. et W.H. MORTON, 1972 - Contribution à l'étude de la grotte de Sof Omar (Ethiopie méridionale). $\mathrm{N}^{\mathrm{O}} 1$ - Introduction. Ann. Spéléol. 27 (1): $105-120$.

PECK, S.B., 1967 - The life cycle of the troglobitic catopid beetle Ptomaphagus loedingi. American Zoologist, 7 (4): 808.

PECK, S.B., 1968 - A new cave Catopid beetle from Mexico with a discussion of its evolution. Psyche, 75 (2): $91-98$.

PESCHILLO, M., 1970 - Morfologia ed evoluzione dell'organo antennale dei Catopidi (Coleoptera). Tesi di Laurea, Ist. Zool., Univ. Roma: $1-197$.

REDDELL, J.R. and R.W. MITCHELL (ed.). 1971 - Studies on the cavernicole fauna of Mexico. Ass. for Mexican Cave Studies, Bull. 4: 1 - 239.

SBORDONI, V. and R. ARGANO, 1972 - Introduction; caves studied during the 1st mission to Mexico (1969). In "Subterranean fauna of Mexico", I. Acc. Naz. Lincei, Quaderno $\mathrm{N}^{\mathrm{O}} 171: 5-22$.

SBORDONI, V., R. ARGANO and A. ZULLINI, 1973 - Biological investigations on the caves of Chiapas (Mexico) and adjacent countries: introduction. In "Subterranean fauna of Mexico", Il. Quaderni Acc. Naz. Lincei, in stampa.

SHEAR, W.A., 1973 - Millipeds (Diplopoda) from mexican and guatemalan caves. In "Subterranean fauna of Mexico", II. Quaderni Acc. Naz. Lincei, in stampa.

VARI AUTORI, 1968 - Mission zoologique belge aux îles Galapagos et en Equador (N. et J. Leleup, 1964 - 1965). I. Mus. R. Afrique Centrale et Inst. R. Sc. Nat. Belgique, Bruxelles: 9 - 272 .

VARI AUTORI, 1970 - Mission zoologique belge aux îles Galapagos et en Equador (N. et J. Leleup, 1964 - 1965). II. Mus. R. Afrique Cen., Inst. R. Sc. Nat. Belgique, Bruxelles: $9-237$.

VARI AUTORI, 1972 - Subterranean fauna of Mexico. I. Acc. Naz. Lincei, Quaderno No171: $3-172$.

VOMERO, V., 1973 - Troglobacanius n.gen., with four new species, a line of mexican troglobitic Histeridae (Ins. Col.). In "Subterranean fauna of Mexico", II. Quaderni Acc. Naz. Lincei, in stampa.

WILKENS, H., 1973 -- Über das phylogenetische Alter von Höhlentieren. Untersuchungen über die cavernicole Süsswasserfauna Yucatans. Z. zool. Syst. Evolut.-forsch. 11: $49-60$.

WILSON, D.E., 1973 - Bat faunas: a trophic comparison. Systematic Zoology, 22 (1): 14-29. 